\title{
Erratum to: A study of vorticity formation in high energy nuclear collisions
}

\author{
F. Becattini ${ }^{1,2, a}$, G. Inghirami ${ }^{1,3}$, V. Rolando ${ }^{4,5}$, A. Beraudo ${ }^{6}$, L. Del Zanna ${ }^{1,2,7}$, A. De Pace ${ }^{6}$, M. Nardi ${ }^{6}$, \\ G. Pagliara $^{4,5}$, V. Chandra ${ }^{8}$ \\ ${ }^{1}$ Dipartimento di Fisica e Astronomia, Università di Firenze, Via G. Sansone 1, 50019 Sesto F.no (Firenze), Italy \\ 2 INFN, Sezione di Firenze, Via G. Sansone 1, 50019 Sesto F.no (Firenze), Italy \\ ${ }^{3}$ Frankfurt Institute for Advanced Studies (FIAS), Johann Wolfgang Goethe University, Frankfurt am Main, Germany \\ ${ }^{4}$ Dipartimento di Fisica e Scienze della Terra, Universitá di Ferrara, Via Saragat 1, 44100 Ferrara, Italy \\ ${ }^{5}$ INFN, Sezione di Ferrara, Via Saragat 1, 44100 Ferrara, Italy \\ ${ }^{6}$ INFN, Sezione di Torino, Via P. Giuria 1, 10125 Turin, Italy \\ ${ }^{7}$ INAF, Osservatorio Astrofisico di Arcetri, L.go E. Fermi 5, 50125 Florence, Italy \\ ${ }^{8}$ Indian Institute of Technology Gandhinagar, Ahmedabad, Gujarat 382424, India
}

Received: 1 March 2018 / Accepted: 12 April 2018 / Published online: 3 May 2018

(C) The Author(s) 2018

Erratum to: Eur. Phys. J. C (2015) 75:406 https://doi.org/10.1140/epjc/s10052-015-3624-1

Due to an oversight of ours in proofreading and a communication problem with the publisher, the figures published in F. Becattini et al. Eur. Phys. J. C (2015) 75:406 were not correct. This Erratum contains the correct figures (Figs. 2, 3, $5,6,7,8,9,10,11,12,13,14,15)$ as in arXiv:1501.04468

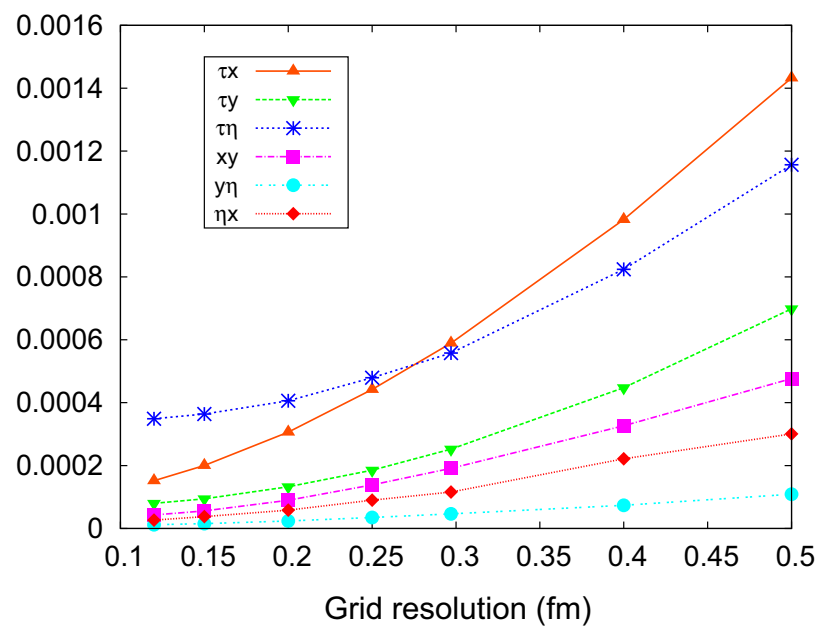

Fig. 2 Mean of the absolute value of T-vorticity components, divided by $T^{2}$, at the freeze-out as a function of the grid resolution

The original article can be found online at https://doi.org/10.1140/ epjc/s10052-015-3624-1. [v2], submitted on March 12 2015, and the post-publication version arXiv:1501.04468 [v3], submitted on August 17 2015.

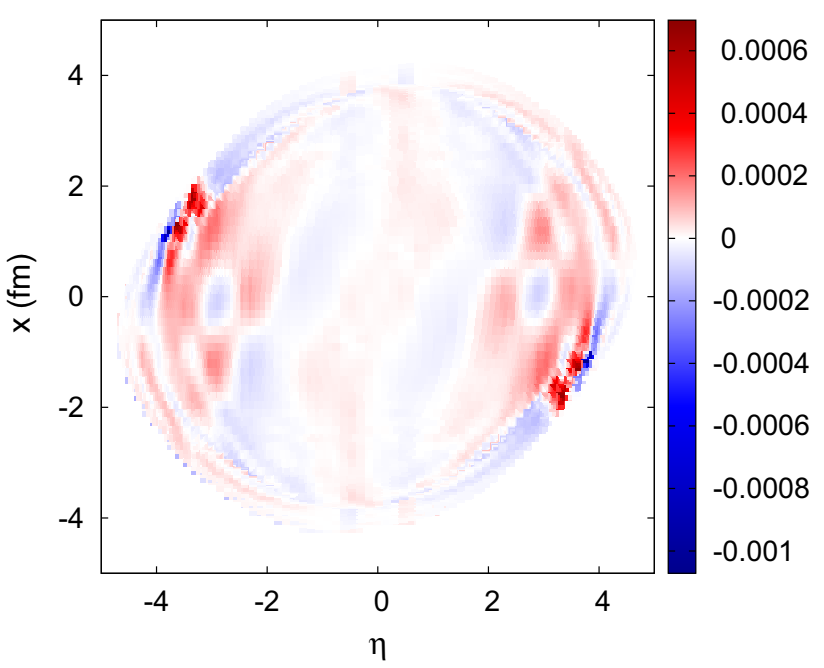

Fig. 3 Contour plot of $\Omega_{x \eta} / \tau T^{2}$ at the freeze-out hypersurface at $y=0$ 

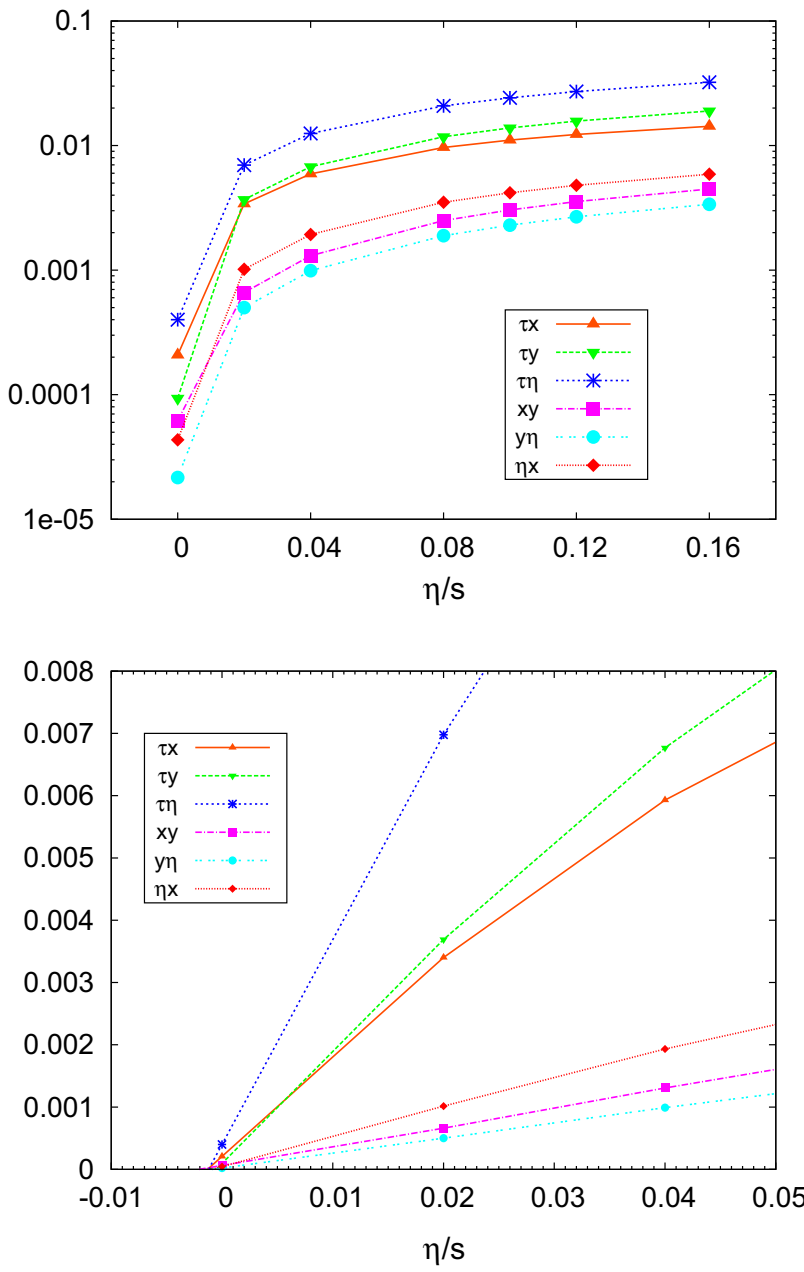

Fig. 5 Mean of the absolute values of $\Omega_{\mu \nu} / T^{2}$ components at the freeze-out hypersurface as a function of $\eta / s$. Note that the $\Omega_{x \eta}, \Omega_{y \eta}, \Omega_{\tau \eta}$ have been multiplied by $1 / \tau$. Upper panel: $\log$ scale. Lower panel: magnification of the region around zero viscosity

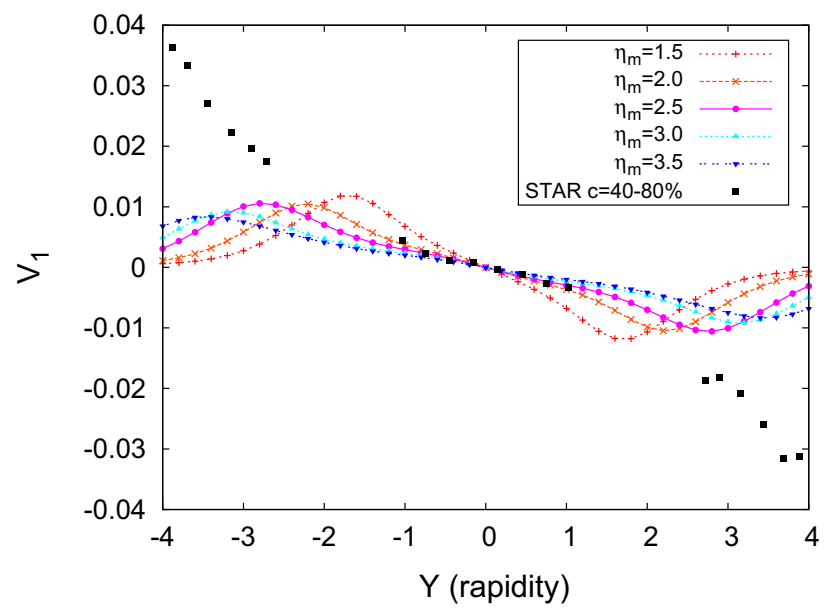

Fig. 6 Directed flow of pions for different values of $\eta_{m}$ parameter with $\eta / s=0.1$ compared with STAR data [1]

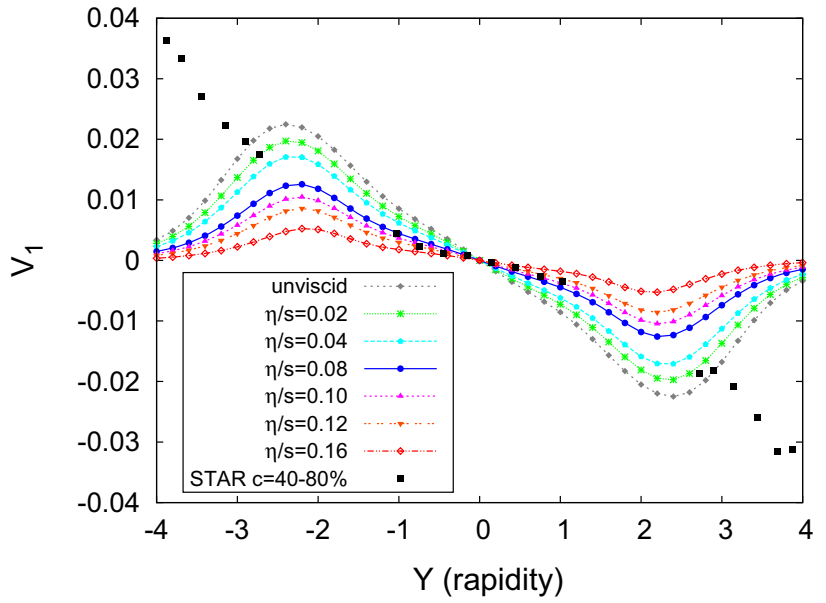

Fig. 7 Directed flow of pions for different values of $\eta / s$ with $\eta_{m}=2.0$ compared with STAR data [1]

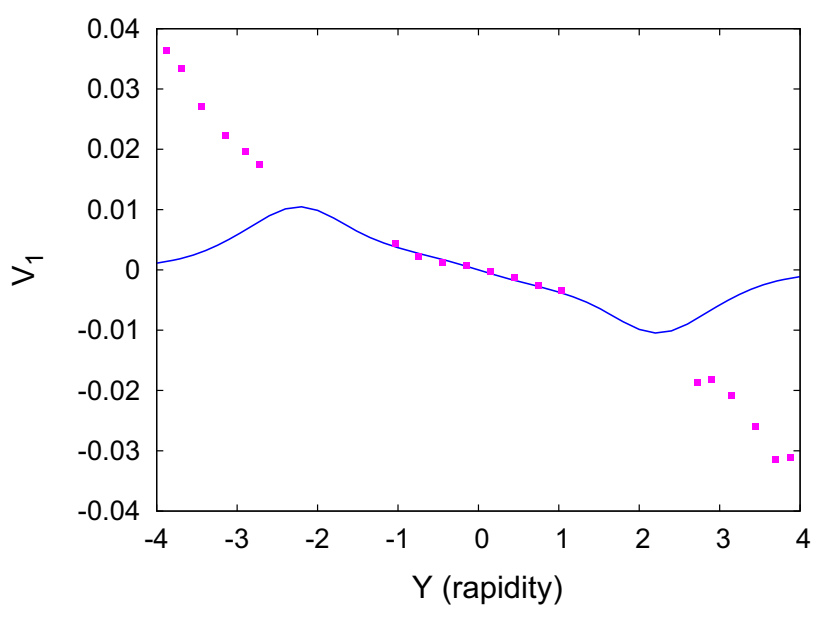

Fig. 8 Directed flow of pions at $\eta / s=0.1$ and $\eta_{m}=2.0$ compared with STAR data [1]

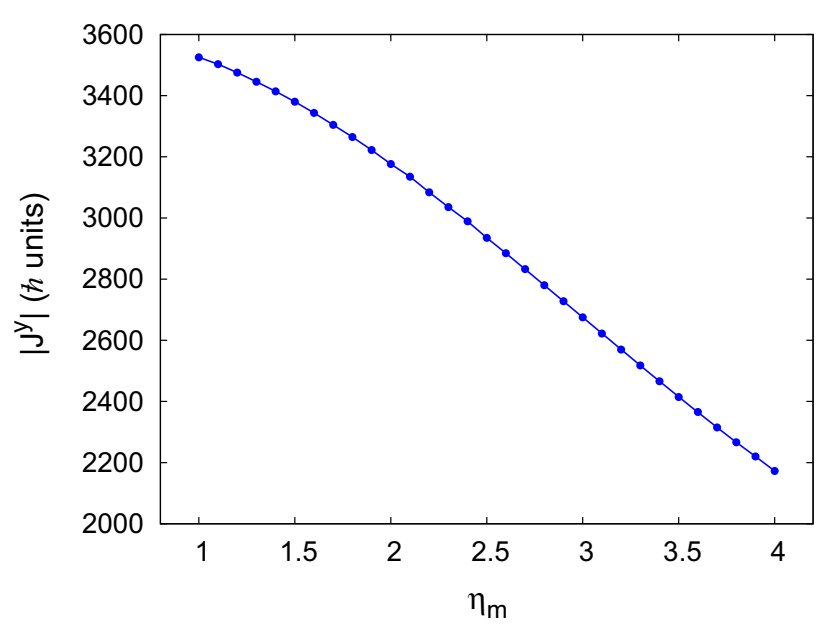

Fig. 9 Angular momentum (in $\hbar$ units) of the plasma with Bjorken initial conditions as a function of the parameter $\eta_{m}$ 


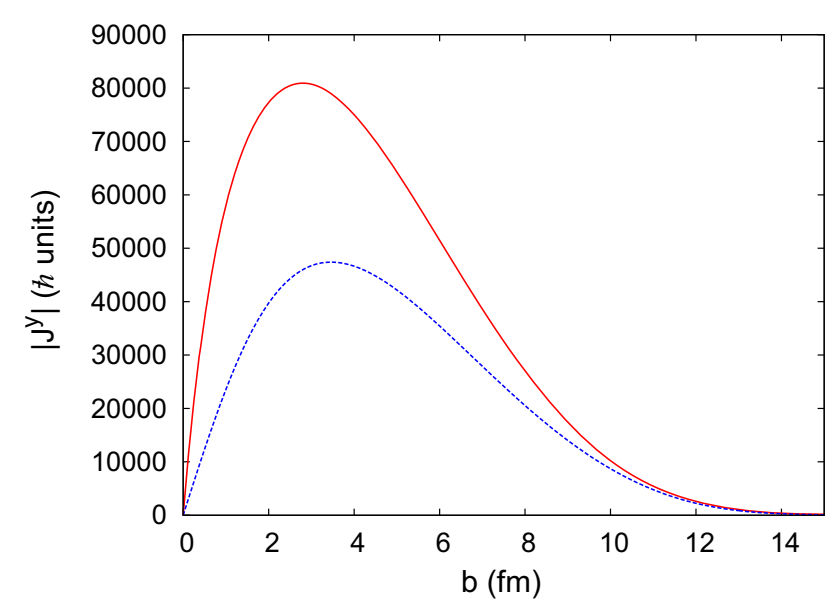

Fig. 10 Estimated angular momentum (in $\hbar$ units) of the overlap region of the two colliding nuclei (solid line) and total angular momentum of the plasma according to the parametrization of the initial conditions (dashed line), as a function of the impact parameter

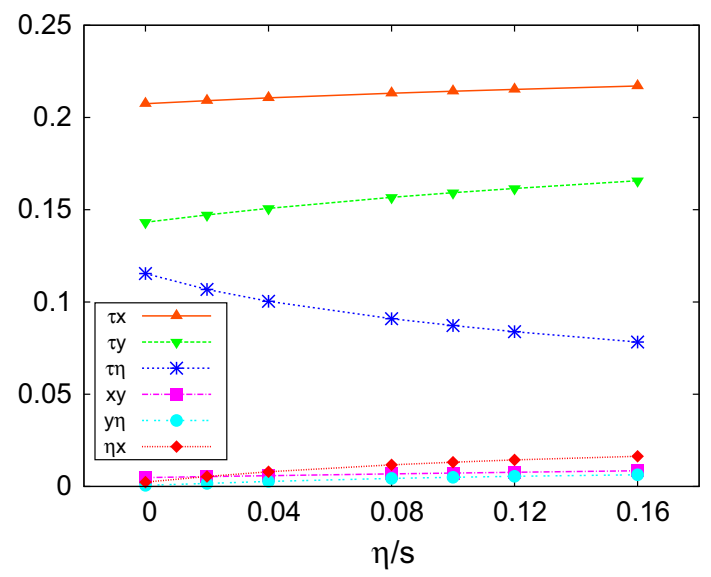

Fig. 11 Mean of the absolute value of thermal vorticity covariant components at the freeze-out as a function of $\eta / s$. Note that the $\varpi_{x \eta}, \varpi_{y \eta}, \varpi_{\tau \eta}$ have been multiplied by $1 / \tau$

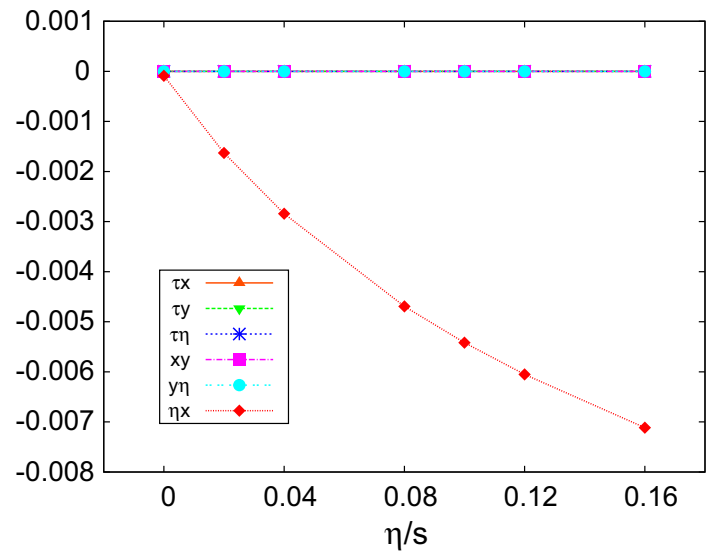

Fig. 12 Mean values of thermal vorticity components at the freeze-out as a function of $\eta / s$. Note that the $\varpi_{x \eta}, \varpi_{y \eta}, \varpi_{\tau \eta}$ have been multiplied by $1 / \tau$

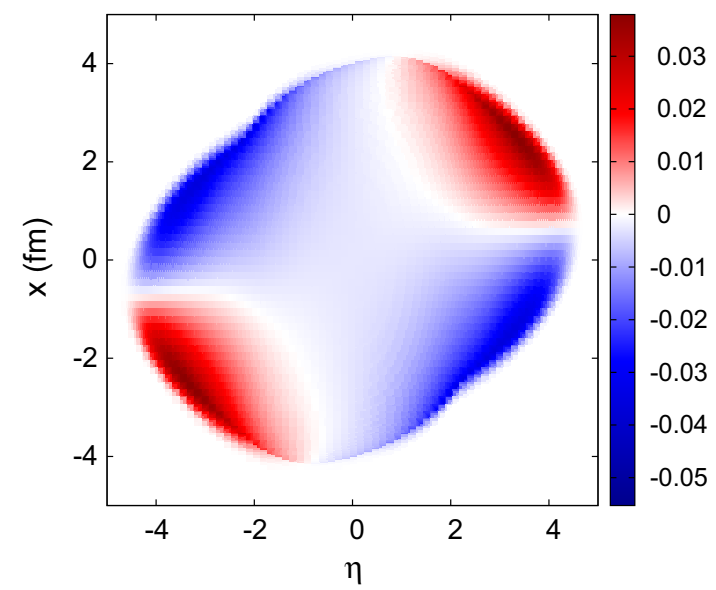

Fig. 13 Contour plot of $1 / \tau$-scaled $\eta x$ covariant component of the thermal vorticity, $\varpi_{\eta x} / \tau$ over the freeze-out hypersurface for $y=0$, $\eta / s=0.1, \eta_{m}=2.0$ 

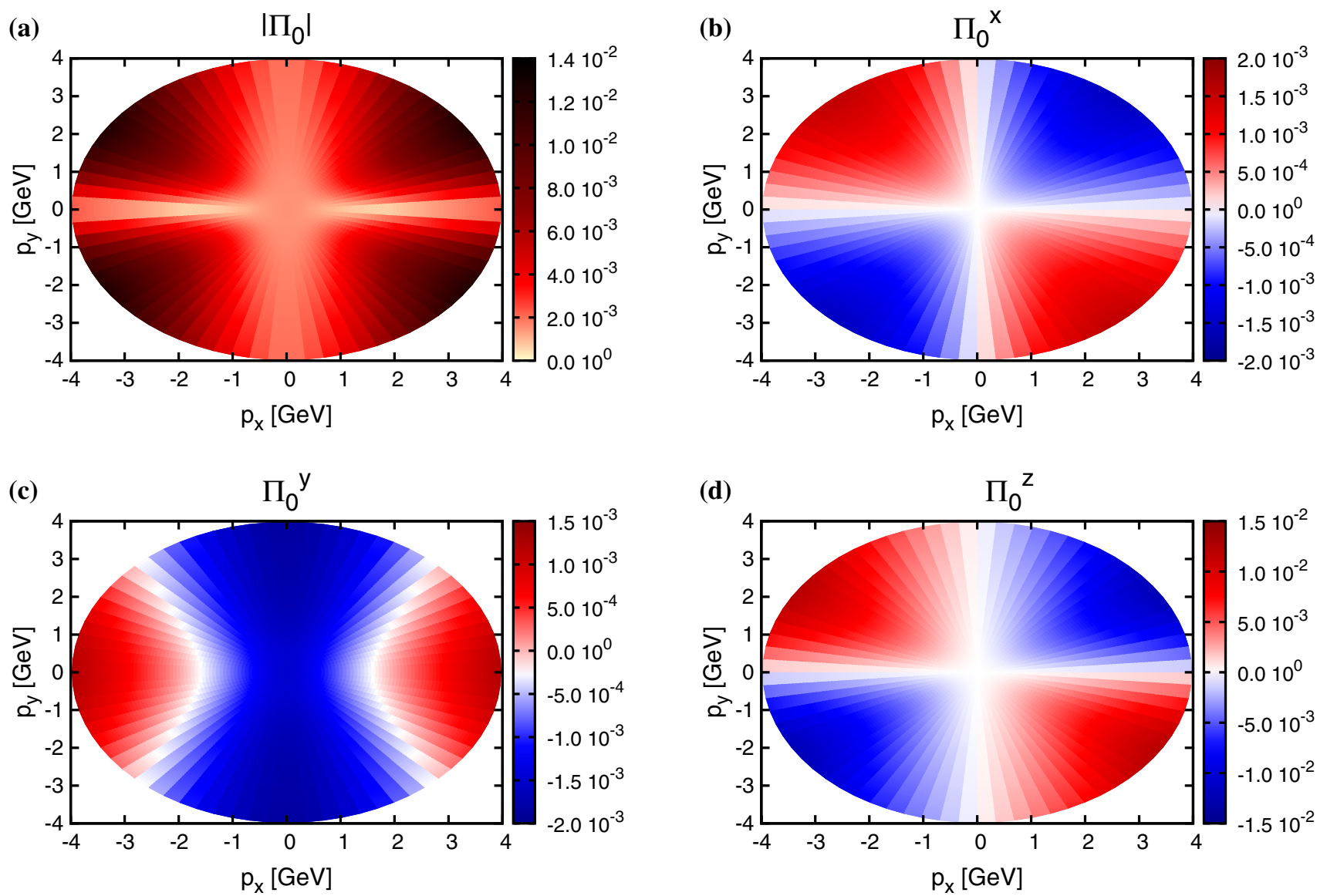

Fig. 14 Magnitude (a) and components (b-d) of the polarization vector of the $\Lambda$ hyperon in its rest frame

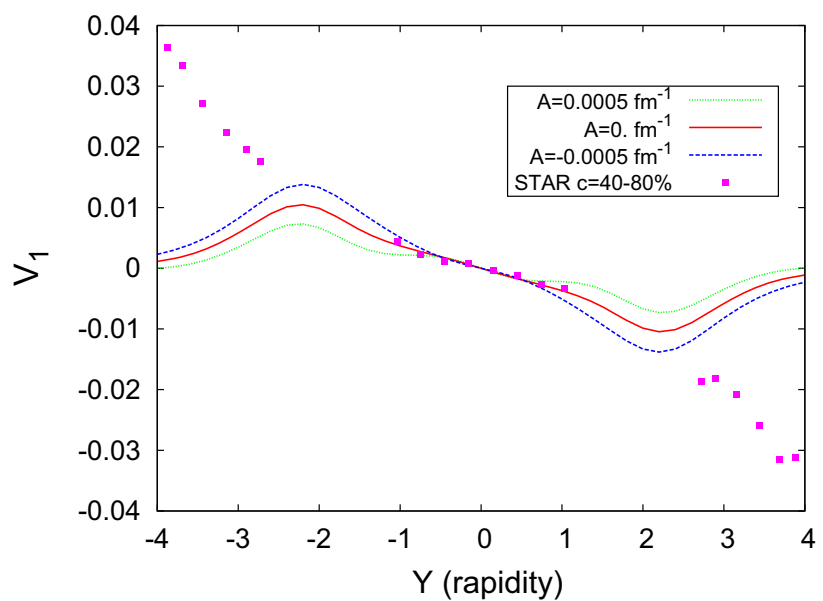

Fig. 15 Directed flow of pions at $\eta / s=0.1$ and $\eta_{m}=2.0$ and with initial $u^{\eta}=\frac{1}{\tau} \tanh A x \sinh \left(y_{\text {beam }}-|\eta|\right)$ as in the eq. (36) of the amended paper (Eur. Phys. J. C (2015) 75:406) compared with STAR data [1]
Open Access This article is distributed under the terms of the Creative Commons Attribution 4.0 International License (http://creativecomm ons.org/licenses/by/4.0/), which permits unrestricted use, distribution, and reproduction in any medium, provided you give appropriate credit to the original author(s) and the source, provide a link to the Creative Commons license, and indicate if changes were made. Funded by SCOAP . $^{3}$

\section{Reference}

1. B.I. Abelev et al. (STAR Collaboration), Phys. Rev. Lett. 101, 252301 (2008) 